\title{
Upregulation of ALDH1B1 promotes tumor progression in osteosarcoma
}

\author{
Xin Wang ${ }^{1, *}$, Yan $\mathbf{Y u}^{2, *}$, Yuting $\mathrm{He}^{2, *}$, Qiqing $\mathrm{Cai}^{1}$, Songtao Gao ${ }^{1}$, Weitao $\mathrm{Yao}^{1}$, \\ Zhiyong Liu ${ }^{1}$, Zhichao Tian ${ }^{1}$, Qicai Han ${ }^{3}$, Weiwei Wang ${ }^{4}$, Ranran Sun ${ }^{2}$, Yonggang \\ Luo $^{3}$ and Chao $\mathbf{L i}^{1}$ \\ ${ }^{1}$ Department of Bone and Soft Tissue, The Affiliated Cancer Hospital of Zhengzhou University, Henan Cancer Hospital, \\ Zhengzhou 450008, China \\ ${ }^{2}$ Precision Medicine Center, The First Affiliated Hospital of Zhengzhou University, Zhengzhou 450052, China \\ ${ }^{3}$ Key Laboratory of Clinical Medicine, The First Affiliated Hospital of Zhengzhou University, Zhengzhou 450052, China \\ ${ }^{4}$ Department of Pathology, The First Affiliated Hospital of Zhengzhou University, Zhengzhou 450052, China \\ *These authors have contributed equally to this work \\ Correspondence to: Chao Li, email: lichao3882496@163.com \\ Yonggang Luo, email: Luoyg_514@126.com
}

Keywords: osteosarcoma; ALDH1B1; progression; proliferation; metastasis

Received: August 21, $2017 \quad$ Accepted: December 04, $2017 \quad$ Published: December 20, 2017

Copyright: Wang et al. This is an open-access article distributed under the terms of the Creative Commons Attribution License 3.0 (CC BY 3.0), which permits unrestricted use, distribution, and reproduction in any medium, provided the original author and source are credited.

\section{ABSTRACT}

Osteosarcoma (OS) is the most common primary malignant bone tumor in childhood and adolescence with poor prognosis. The mechanism underlying tumorigenesis and development of OS is largely unknown. ALDH1B1 has been reported to involve in many kinds of human cancers and functions as an oncogene, but the role of ALDH1B1 in OS has not been investigated comprehensively. In the present study, we aimed to examine clinical value and biological function of ALDH1B1 in OS. Firstly, we investigated the roles of ALDH1B1 on an OS tissue microarray (TMA) as well as two OS cohorts from GEO database. We found that ALDH1B1 was significantly up-regulated in OS tissues and was independently associated with poor prognosis. Moreover, ALDH1B1 silencing could suppress the proliferation, migration, invasion in vitro and inhibit the growth of xenograft tumor and of OS cells in vivo. Additional, ALDH1B1 knockdown increased the apoptosis rate and lead to cell cycle arrest in $\mathbf{G 1}$ stage of OS cell in vitro. More importantly, the inhibition of ALDH1B1 expression could increase the sensitivity of OS cells to chemotherapy, which suggested that ALDH1B1 might be served as a therapeutic target to reverse drug resistance in chemotherapy in OS patients. Taken together, our founding suggested that ALDH1B1 contributes to OS tumor progression and drug resistance, which may represent a novel prognostic marker and potential therapeutic target for OS patients.

\section{INTRODUCTION}

Osteosarcoma (OS) is the most common primary malignant bone tumor in childhood and adolescence [1]. The incidence is about $1 \sim 3 / 1000,000$ per year throughout the world, and accounts for $5 \%$ of all pediatric malignancies, with highly aggressive and early systemic metastasis [1-4]. The combination of surgery resection and multi-chemotherapy has become a standard treatment strategy for almost all OS patients [5,6]. The prognosis of OS patients remains unsatisfactory due to bone destruction, lung metastasis and chemotherapy resistance [7-9]. Currently, the underlying molecular mechanism which involved in OS origination, metastasis and chemoresistance has not been fully elucidated [10]. Therefore, a better understanding of the molecular biology of osteosarcoma is needed and may improve therapeutic efficiency and clinical outcomes for OS patients [5, 6]. 
There are 19 aldehyde dehydrogenases (ALDHs) in human body [11]. Abnormal expression and unbalanced biological activity of ALDHs are related with a variety of diseases [12]. Aldehyde dehydrogenase 1 (ALDH1), a subfamily of ALDH, is composed of six enzymes (ALDH1A1, ALDH1A2, ALDH1A3, ALDH1B1, ALDH1L1 and ALDH1L2) that are highly expressed in stem cells and regulate the stem cell function [11, 13]. Increased ALDH1 activity has been found in the stem cell populations of human acute myeloid leukemia, multiple myeloma, and a number of solid tumors [1417]. Therefore, recent studies have focused on revealing which ALDH isoforms and how these may contribute to the progression of cancer. Of these, ALDH1A1 plays a crucial role in the development of colorectal cancer, non-small cell lung cancer, ovarian cancer and EpsteinBarr virus-associated nasopharyngeal carcinoma [1821]. In addition, ALDH1A3 is more widely expressed and associated with poor survival in neuroblastoma [22]. ALDH1A3 and ALDH1L1 are found to be significantly correlated to the worsened overall survival for all patients with gastric cancer and are potential prognostic markers and therapeutic targets for patients with gastric cancer [23]. Therefore, all of these studies make ALDH1 family as a potential predictive biomarker of cancers.

Recent studies have revealed that ALDH1B1, another number of ALDH1 family, plays a vital role in several human cancers. It has been confirmed that ALDH1B1 was up-regulated in several cancers such as colorectal cancer, pancreatic adenocarcinoma, non-smallcell lung cancer and gastric cancer, and it is identified to be involved in tumorigenesis and metastasis. Additional, up-regulated expression of ALDH1B1 was associated with poor prognosis and clinical malignancies [24-27]. However, the expression and functional role of ALDH1B1 in OS are still unclear.

In the present study, to determine ALDH1B1 expression and investigated the roles of ALDH1B1 in OS, we firstly analyzed the expression of ALDH1B1 in an OS tissue microarray (TMA) contains 40 clinically annotated OS tissues through immunohistochemical (IHC). We found that ALDH1B1 was markedly upregulated and associated with poor prognosis in OS patients. Additional, two OS gene expression cohorts from Gene Expression Omnibus (GEO) database were employed to validate the results of TMA. ALDH1B1 silencing led to inhibit cell proliferation, migration and invasion while promote apoptosis and cell cycle arrest in vitro. Meanwhile, downregulation of ALDH1B1 inhibited the tumor growth in vivo. Moreover, the inhibition of ALDH1B1 expression could increase the sensitivity of OS cells to chemotherapy. In summary, our results suggest that ALDH1B1 can be considered as a potential prognostic marker and therapeutic target for OS patients, especially for patients with metastasis or drug resistant to chemotherapy.

\section{RESULTS}

\section{High expression of ALDH1B1 was closely correlated with prognosis of OS patients}

To determine the clinical significance of ALDH1B1 in OS, the correlation of ALDH1B1 expression and survival outcome in 40 osteosarcoma patients were analyzed. Firstly, TMA were constructed and the protein expression of ALDH1B1 was analyzed by IHC. ALDH1B1 staining was scored ranged from score $1+$ to score 5+, and the details were shown in Figure 1A. There was no difference between high ALDH1B1 expression (score 4+ and 5+) and low ALDH1B1 expression (score $1+, 2+$ and $3+$ ) patients in age, gender distribution, tumor site and tumor recurrence by Pearson's $\chi 2$ test or Fisher's exact test analysis (all $\mathrm{P}>0.05$ ). However, the expression of ALDH1B1 were closely related with tumor TNM stage $(\mathrm{P}=0.027)$, tumor size $(\mathrm{P}=0.001)$, tumor metastasis $(\mathrm{P}=0.006)$ and patients survival $(\mathrm{P}=0.001)$ (Table 1$)$. More importantly, we found that patients with high ALDH1B1 expression possessed a poor survival outcome compared with the patients with low expression of ALDH1B1 by Kaplan-Meier survival analysis (Log-rank tests, $\mathrm{P}=0.004$ ) (Figure $1 \mathrm{~B}$ ). In addition, a high score of ALDH1B1 staining was determined in patients with TNM III versus the patients with TNM II $(\mathrm{P}=0.0291)$ and TNM I $(\mathrm{P}=0.0076$, Figure $1 \mathrm{C})$. Our patients' results indicated that high expression of ALDH1B1 was closely correlated with poor prognosis of OS patients.

\section{High expression of ALDH1B1 mRNA in OS tissues predicted a poor prognosis in patients by GEO database}

To further confirm the correlation between ALDH1B1 expression and the prognosis of OS patients, GEO database of OS (GSE21257 and GSE39055) was used. After surgery, patients with high ALDH1B1 expression possessed a poor survival outcome compared with the patients with low expression of ALDH1B1 by Kaplan-Meier survival analysis (GSE21257: Log-rank tests, $\mathrm{P}=0.0396$; GSE39055: Log-rank tests, $\mathrm{P}=0.0416$ ) (Figure 2A\&2B), consistent with our patients' results. Moreover, patients with metastasis presented a high ALDH1B1 expression compared with the patients without metastasis $(\mathrm{P}=0.0344)$ (Figure 2C). These data proved that the high expression of ALDH1B1 could be served as a biomarker to predict the prognosis of OS patients.

\section{High expression of ALDH1B1 was crucial for the growth and proliferation, invasion and migration, apoptosis and cell cycle of OS cells}

To evaluate the clinical significance and function of ALDH1B1 in OS, we firstly detected the expression 
Table 1: Correlation of clinico-pathological features with ALDH1B1 expression levels in 40 OS patients

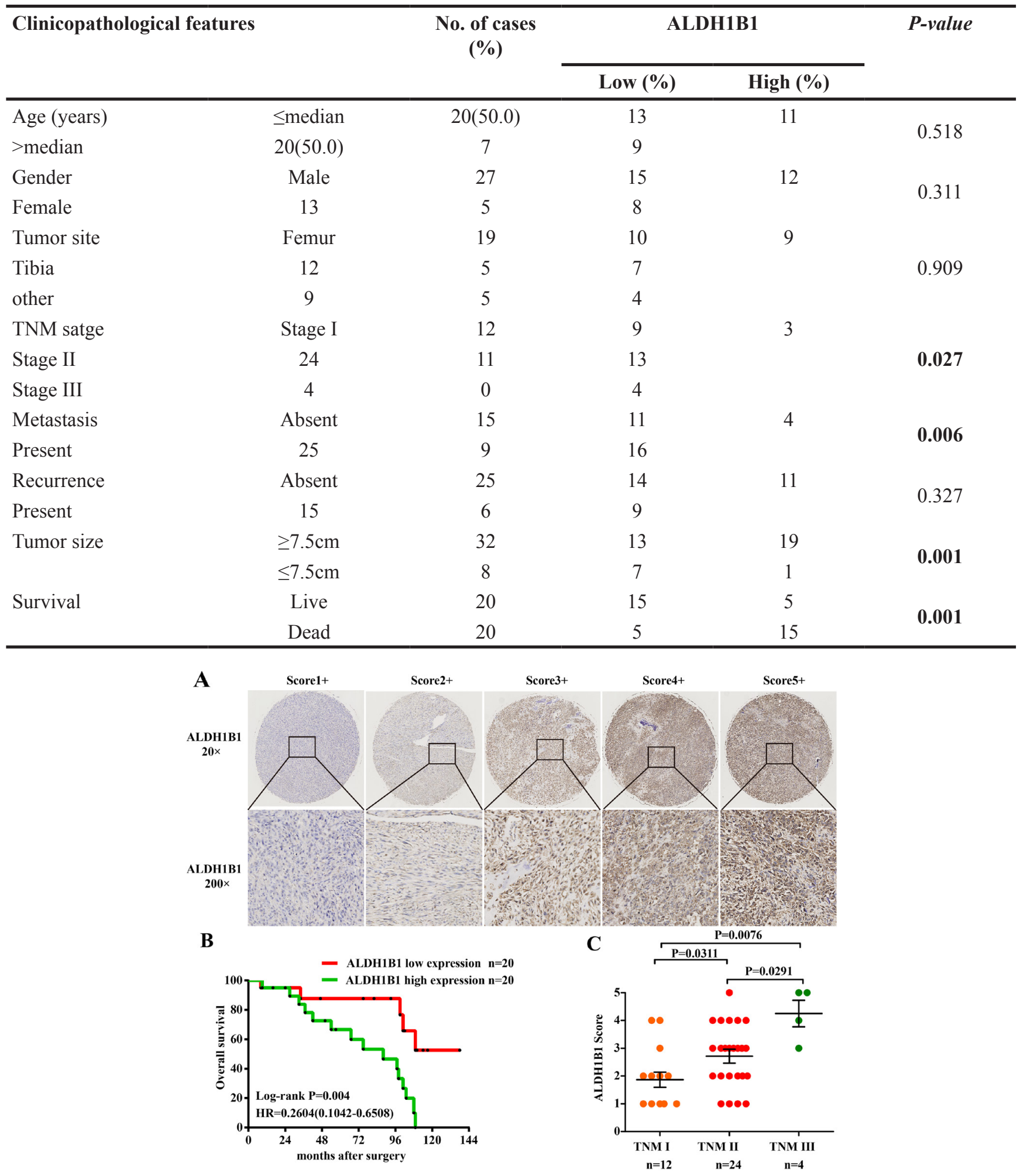

Figure 1: Association of ALDH1B1 expression and clinical significance in our OS patients. (A) Representative images of different immunohistochemical staining intensities of ALDH1B1 are shown in OS tissues. The percentage of cells showing positive staining was calculated by reviewing the entire spot. On the basis of the percentage, the staining patterns were categorized into 5 groups: $1,1+$, positive cells $<10 \% ; 2,2+$, positive cells $10 \%$ to $25 \% ; 3,3+$, positive cells $26 \%$ to $50 \%$; 4 , 4+, positive cells $51 \%$ to $75 \%$; and $5,5+$, positive cells $>75 \%$. (Original magnification, $\times 400$ ). (B) Kaplan-Meier analysis was used to analyzed the overall survival of OS patients with low ALDH1B1 expression (score 1+, 2+ and 3+) and high ALDH1B1 expression (score 4+ and 5+). (C) Distribution of ALDH1B1 staining scores among the patients with TNM I, II and III stages. 
A

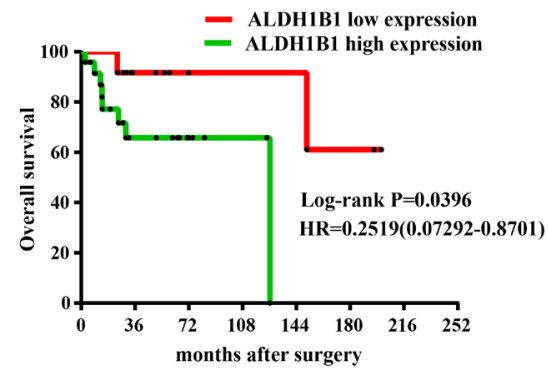

B

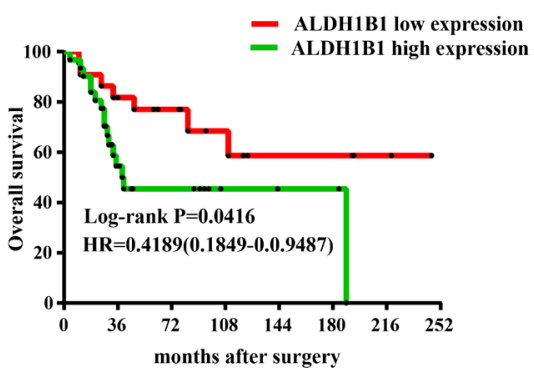

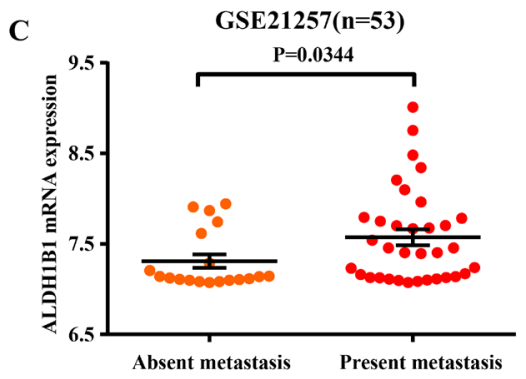

Figure 2: Association of ALDH1B1 expression and prognosis of OS patients by analyzing the GEO database. Kaplan-Meier were used to analyzed the overall survival of OS patients with low ALDH1B1 expression and high ALDH1B1 expression in GSE21257 (A) and GSE39055 (B). (C) Distribution of ALDH1B1 staining scores among the patients with metastasis and without metastasis in GSE21257.

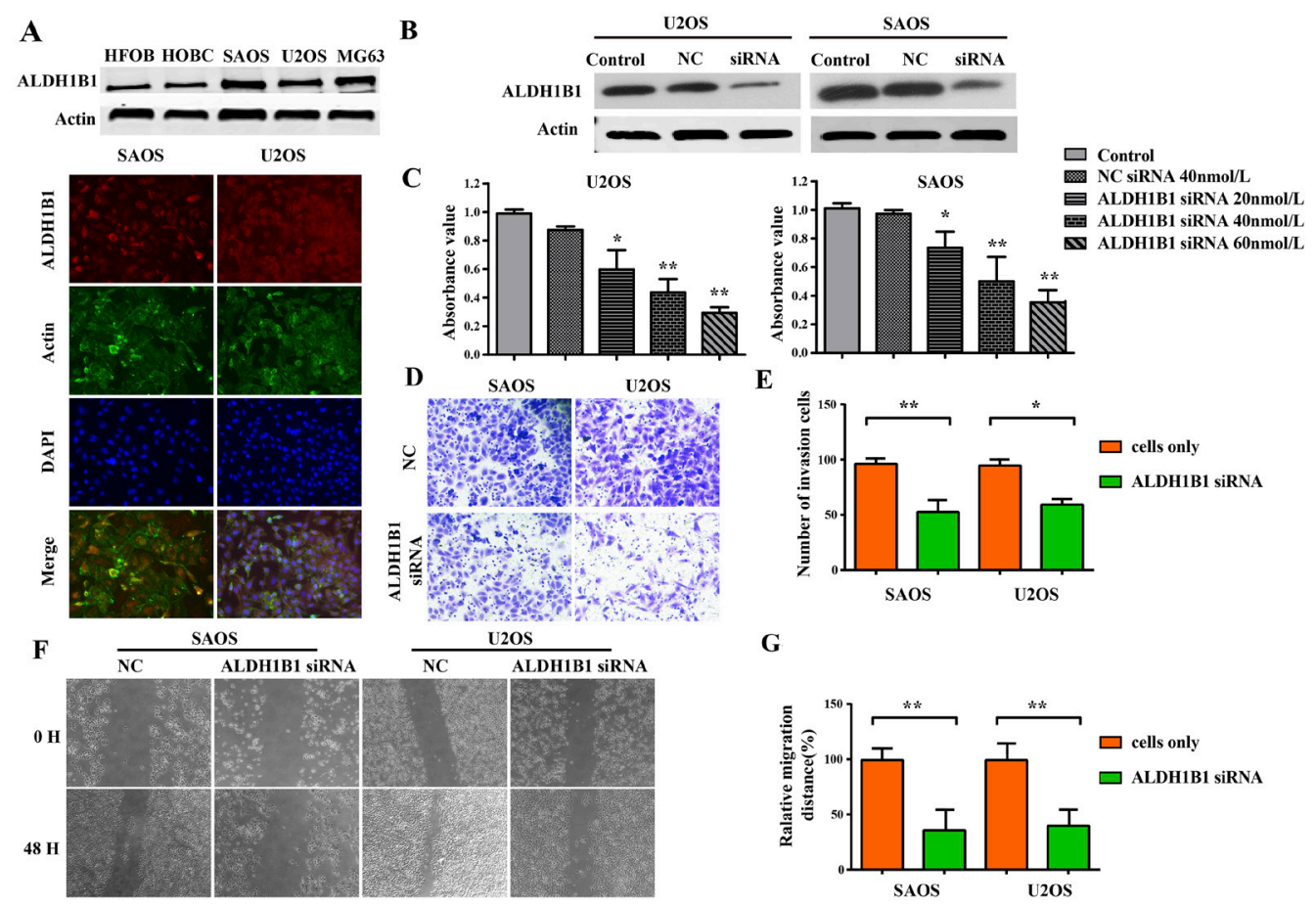

Figure 3: The role of ALDH1B1 in the growth and proliferation, invasion and migration of OS cells. (A) The expressions of ALDH1B1 in OS cell lines (SAOS, U2OS and MG63) and normal human osteoblast cell lines (HFOB and HOBC) were determined by western blot and Immunofluorescence assay. (B) Downregulated expression of ALDH1B1 by synthetic siRNA in SAOS and U2OS cell lines under the different ALDH1B1 siRNA concentrations (20, 40 and $60 \mathrm{nmol} / \mathrm{L})$. (C) Downregulated expression of ALDH1B1 inhibited the proliferation of OS cells by MTT assay. Downregulated expression of ALDH1B1 inhibited the invasion of OS cells by transwells assay (D, E). Downregulated expression of ALDH1B1 inhibited the migration of OS cells by wound healing assay (F, G). 
of ALDH1B1 protein in OS cell lines by western blotting. We found that ALDH1B1 was significantly increased in OS cell lines (MG63, U2OS and SAOS) compared with in normal human osteoblast cell lines (HFOB and HOBC) (Figure 3A). Meanwhile, immunofluorescence analysis of ALDH1B1 was performed and ALDH1B1 was positive in OS lines (U2OS and SAOS) (Figure 3A). Then, ALDH1B1 siRNA was transfected in the U2OS and SAOS cell lines, respectively. The non-specific siRNA (NS siRNA) was served as the control. The efficacy of siRNA was confirmed by western blot analysis (Figure 3B). Along with the increase of ALDH1B1 siRNA concentration (from 15 to $45 \mathrm{nmol} / \mathrm{L}$ ), ALDH1B1 expressions were gradually decreased in both U2OS and SAOS cell lines (Figure 3C).
Malignant tumors are characterized by uncontrolled cell proliferation and growth, activating invasion and metastasis [28]. We evaluated cells proliferation by MTT assay and EDU assay, and found that cells proliferation was inhibited obviously in the U2OS and SAOS cell lines after ALDH1B1 siRNA administration compared with the NS siRNA administration (Figure 3D, 4F\&4G). Moreover, the invasion and migration ability of OS cells after ALDH1B1 siRNA administration were examined by the matrigel invasion assay and wound healing assay. As shown in the matrigel invasion assay, the invasion cells of OS cells after ALDH1B1 siRNA administration were obviously decreased compared with that after the NS siRNA administration $(\mathrm{P}<0.01)$ (Figure $3 \mathrm{E} \& 3 \mathrm{~F})$. In the wound healing assay, we found that the
A

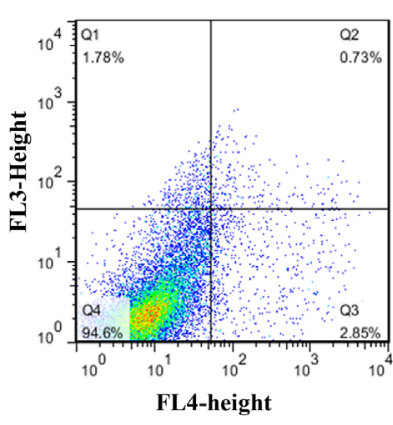

C

U2OS-NC
U2OS-ALDH1B1 SIRNA

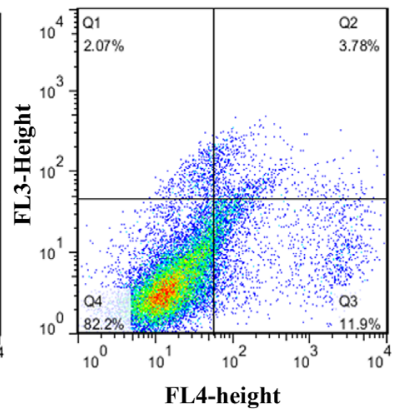

U2OS-. ILDH1B1 siRNA
B

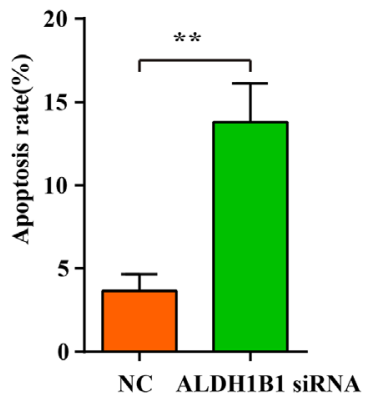

D

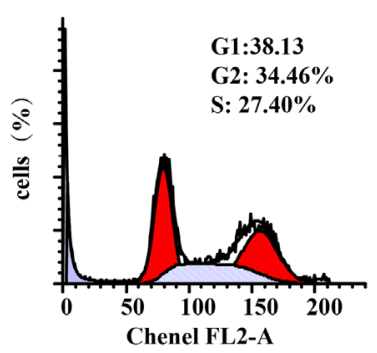

Chenel FL2-A
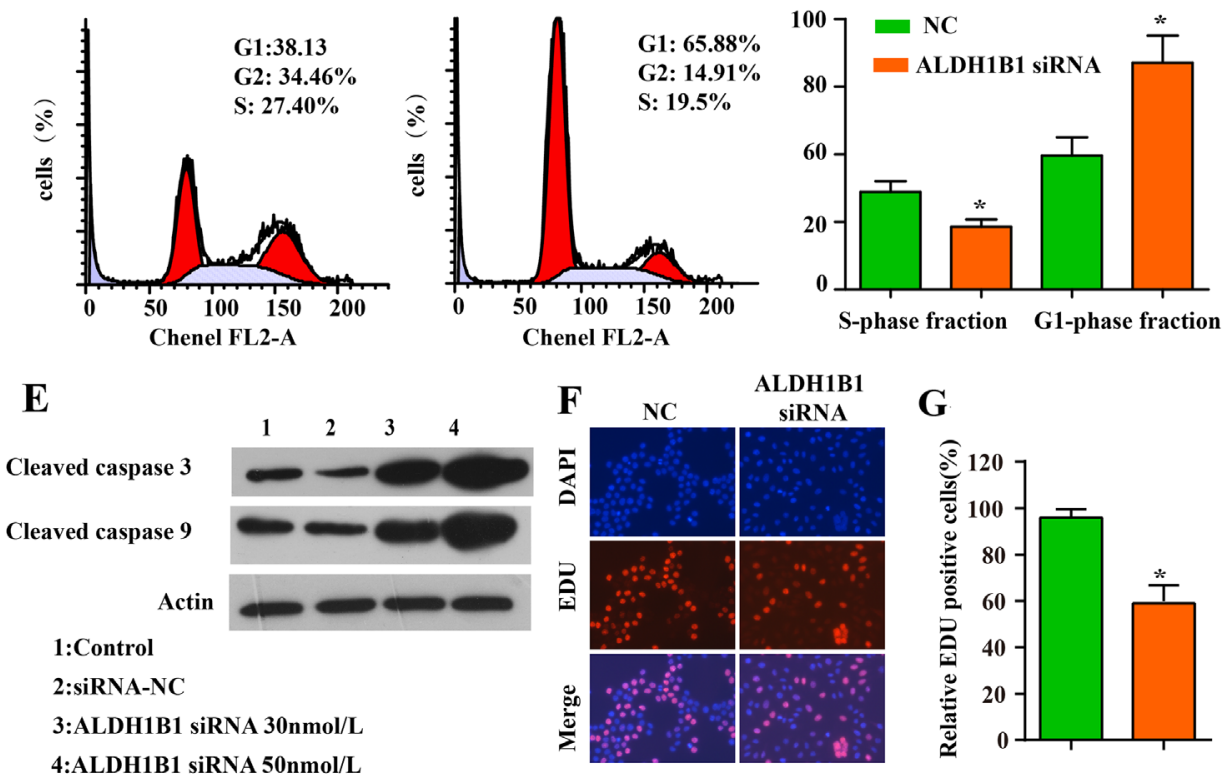

Figure 4: The role of ALDH1B1 in the apoptosis, cell cycle of OS cells. Downregulated expression of ALDH1B1 promoted the apoptosis of OS cells by flowcytometry assay (A, B). Downregulated expression of ALDH1B1 led to the cell cycle arrest in G1 phase, resulting in a decrease rate of S phase (C, D). (E) Downregulated expression of ALDH1B1 induced the activity of apoptosis-related proteins (cleaved caspase-3/9) in a dose-dependence way. (F, G) Downregulated expression of ALDH1B1 inhibited the growth and proliferation of OS cells. 
relative migration distance were obviously decreased in OS cells with ALDH1B1 siRNA, compared to the blank control and NS siRNA treated cells $(\mathrm{P}<0.01)$ (Figure 3G). Thus, both wound healing assay and matrigel invasion assay revealed that the inhibition of ALDH1B1 expression by siRNA transfection significantly suppressed the migration and invasion of osteosarcoma cells. Currently, apoptosis induced programmed cell death and controlled cell cycle are considered as a natural barrier to cancer pathogenesis [28]. To determine whether ALDH1B1 was involved in the regulation of apoptosis, the rate of apoptosis and the activity of apoptosis-related proteins was determined in OS cells after ALDH1B1 siRNA administration. We found that ALDH1B1 siRNA administration increased the apoptosis rate and the levels of cleaved caspase- 3 and 9 (Figure 4A, 4B\&4E). Moreover, cell cycle was observed and we found that inhibition of ALDH1B1 expression led to cell cycle arrest in G1 phase, resulting in a decreased rate of $\mathrm{S}$ phase (Figure 4C\&4D). These results indicated that high expression of ALDH1B1 prompted the growth and proliferation, invasion and migration and cell cycle, while inhibited apoptosis of OS cells.

\section{Inhibition of ALDH1B1 suppressed drug resistance and stemness of OS cells}

Recent studies have proved that ALDH1A1 is linked to drug resistance in chemotherapy [29, 30]. With a similar role of ALDH1A1 in cancer, we also found that the inhibition of ALDH1B1 expression could increase the sensitivity of OS cells to chemotherapy (doxorubicin) (Figure 5A), which were further supported by the inhibition abilities of colony forming and proliferation after ALDH1B1 silencing in the presence of doxorubicin
A

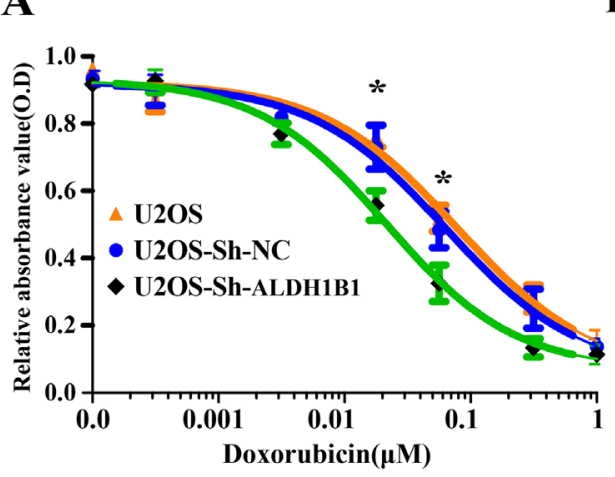

D

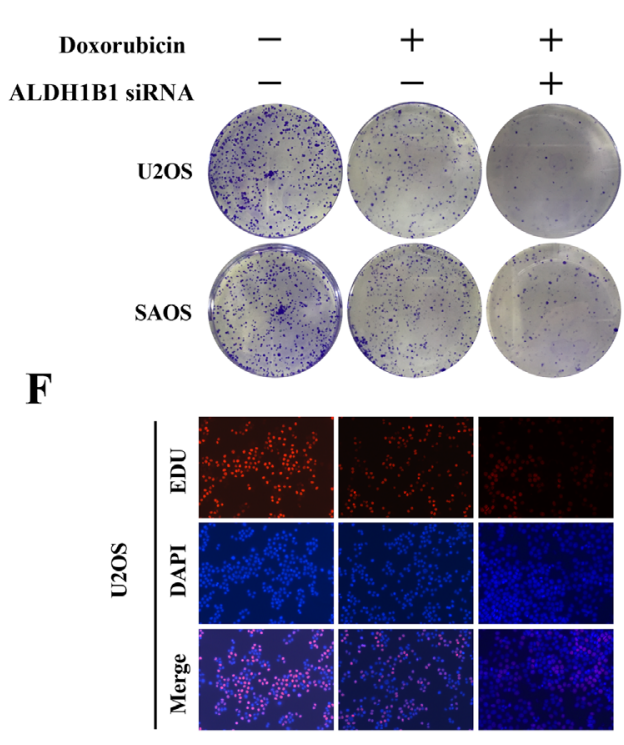

B

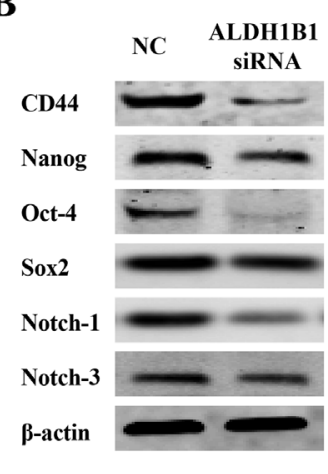

E
C

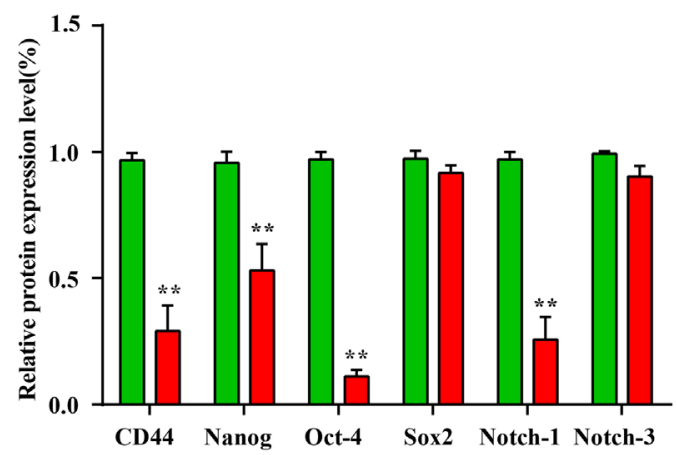

G
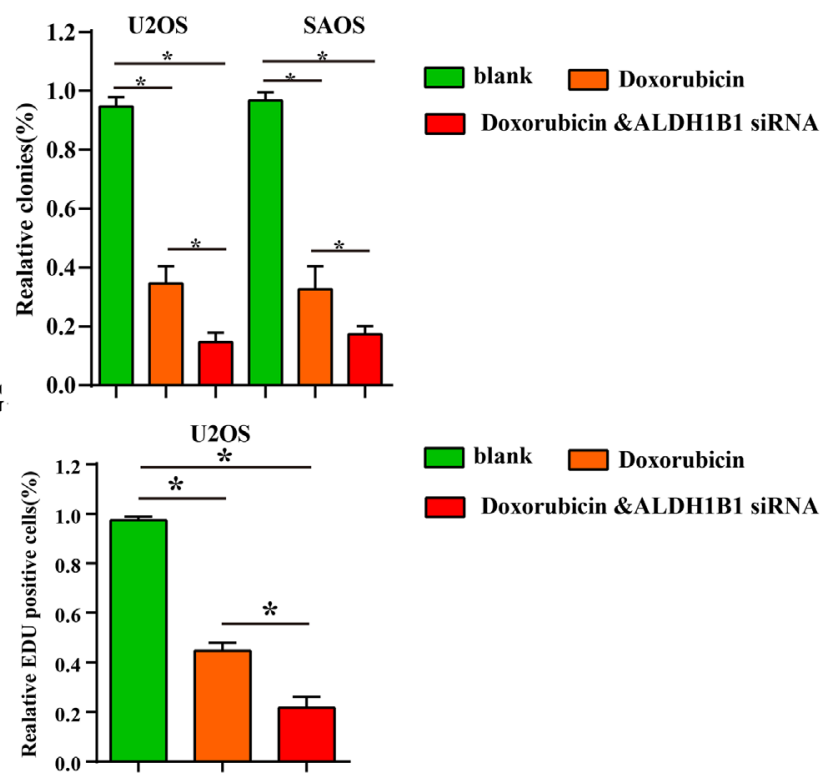

Figure 5: The role of ALDH1B1 in drug resistance and stemness of OS cells. (A) Downregulated expression of ALDH1B1 increased the cell sensitivity of chemotherapy (doxorubicin) by drug sensitivity assay. In the presence of doxorubicin, downregulated expression of ALDH1B1 further inhibited the colony formation (D, E) and proliferation abilities of OS cells (F, G). The role of ALDH1B1 on the protein expressions of cancer stem cell markers, including CD44, Nanog, Oct-4 and Notch-1 (B, C). 
(Figure 5D-5G). Given the activity of ALDH family is a marker of CSCs in many solid cancers and CSCs are a risk factor for carcinogenesis and responsible for tumor initiation, growth, metastasis [31], we examined the effect of ALDH1B1 silencing on the protein expressions of cancer stem cell markers, including Nanog, Sox2, Oct-4, CD44, Notch-1 and Notch-3, in osteosarcoma cells. As shown in Figure 5B\&5C, the expressions of CD44, Nanog, Oct-4 and Notch-1 were remarkably inhibited in OS cells. Taken together, our data indicated that ALDH1B1 played a crucial role in drug resistance and stemness of OS cells.

\section{ALDH1B1 silencing inhibits the tumor growth of OS in vivo}

The function of ALDH1B1 was further elucidated in vivo by subcutaneous tumor transplantation with the control vector, NC shRNA, ALDH1B1 shRNA. The inhibition of ALDH1B1 was confirmed by western blot and IHC (Figure 6A\&6F). We found that the silencing of ALDH1B1 inhibited the tumor growth of OS (Figure 6B-6E). Meanwhile, the expression of Ki-67, a nuclear protein that is associated with cell proliferation, was decreased after ALDH1B1 silencing (Figure 6F). These results indicated that high expression of ALDH1B1 inhibited tumor growth in vivo, probably by inhibiting cell proliferation in OS.

\section{DISCUSSION}

OS is the most common primary malignant bone tumor in childhood and adolescence, with highly aggressive and early systemic metastasis [1-4]. Even the development of therapeutic strategies, there are only less than $30 \%$ of OS patients with metastasis who can survive with disease-free [7, 8, 32, 33]. Meanwhile, chemotherapy resistance is also a significant obstacle for OS treatments [34]. Thus, a better understanding of the molecular biology of OS is needed and may improve therapeutic efficiency and clinical outcomes for patients with osteosarcoma $[5,6]$.

There are 19 ALDHs in human body [11]. Abnormal expression and unbalanced biological activity of ALDHs are related with a variety of disease, including alcoholic liver disease, Sjögren-Larsson syndrome, type 2 hyperprolinemia, hyperammonemia, Parkinson's disease and cancers [12]. Referring to ALDH1 subfamily, recent studies have reported that ALDH1A1 and ALDH1A3 were significantly correlated to the poor overall survival and were considered as potential prognostic markers and therapeutic

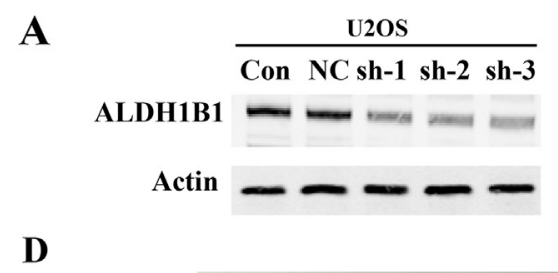

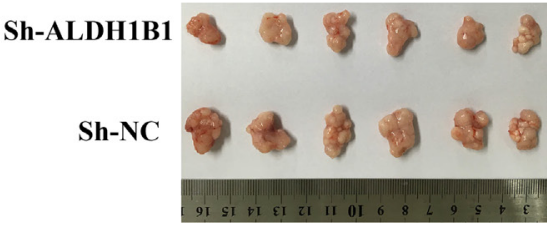

$\bigcirc_{\text {Sh-ALDH1B1 }}^{\text {Sh-NC }}$

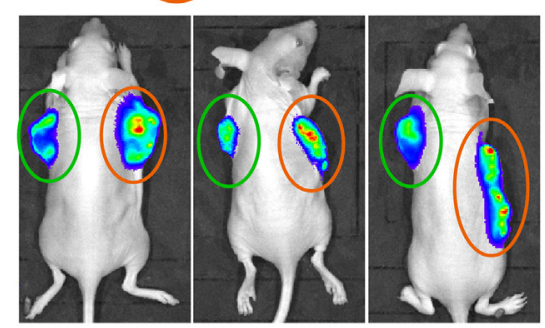

$\mathbf{F}$

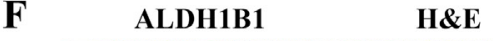

C

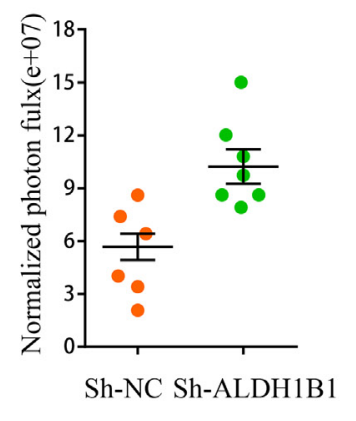

Ki-67

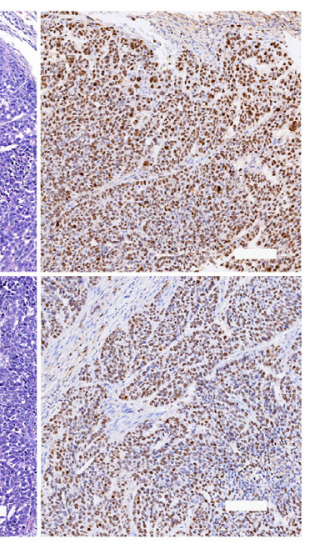

Figure 6: The roles of ALDH1B1 in OS in vivo. (A) Downregulated expression of ALDH1B1 in OS cells via shRNA was confirmed by western blot. The tumor growth was detected by In-Vivo Imaging Systems (B, C) and by tumor volumes (D, E). (F) Immunohistochemistry staining of ALDH1B1 and Ki67 in OS tissues from nude mice. 
targets for patients $[22,23,29,30,35]$. ALDH1B1 is an enzyme to compose of ALDH1, a subfamily of ALDHs. It has been previously shown that ALDH1B1 could promote colon cancer tumorigenesis by modulating the Wnt/ $\beta$-catenin, Notch and PI3K/Akt signaling pathways and might be considered as a selective therapeutic targets to prevent or treat colon cancer $[26,36]$. In pancreatic adenocarcinoma, ALDH1B1 is over-expressed and may be an critical modulator of tumor progression. ALDH1B1 silencing could inhibit growth of tumors in vitro and vivo [25]. However, the expression and function of ALDH1B1 in OS has not been well characterized. In the present study, we identified a novel oncogenitc function of ALDH1B1 in OS. Consistent with the result confirmed by above studies in other cancers, we firstly confirmed that ALDH1B1 was significantly over-expressed in OS tissues and cell lines. Furthermore, the high ALDH1B1 expression was significantly correlated with overall survival, tumor metastasis and TNM stages of osteosarcoma patients. These results indicated that ALDH1B1, similar with other numbers of ALDH family [18-22], also could be considered as a potential prognostic marker.

Given the observed up-regulated ALDH1B1 expression in OS, we next determined whether ALDH1B1 be able to modulate proliferation of OS cells. Result shown that the inhibition of ALDH1B1 expression could suppress the growth/proliferation, migration, invasion in vitro. Meanwhile ALDH1B1 silencing could promote apoptosis and lead to cell cycle arrest in G1 stage of OS cell in vitro. In addition, the silence of ALDH1B1 inhibited the tumor growth in vivo, probably by inhibiting cell proliferation in OS. These results suggest that ALDH1B1 may contribute to the growth of OS cells which possess high expression levels of ALDH1B1. However, the mechanism underlying by which ALDH1B1 silencing affect the cell proliferation ability remains to be established.

It has been well-established that biological activity of ALDH family is a marker of cancer stem cells (CSC) in many solid cancers [37-39]. Cancer stem cells have been implicated in the tumor formation and in the development of chemotherapy resistance in a number of malignances [40]. In light of the crucial role of CSC in the progression in cancers, it's not a surprise that ALDH family could contribute to tumorigenesis. To confirm our speculation, we investigated whether ALDH1B1 facilitate the tumor growth by regulating stemness of OS in a subsequent mechanistic experiment. We examined the effect of ALDH1B1 silencing on the protein expressions of cancer stem cell markers. As shown in Figure 5E and 5F, the expressions of CD44, Nanog, Oct-4 and Notch-1 were remarkably inhibited in OS cells. Taken together, our data indicate that ALDH1B1 played a crucial role in OS cell proliferation, at least in part by modulating cancer stem function. Additional, ALDH1B1, an alcohol metabolism associated enzyme located in mitochondrial, may affect cellular metabolism by which facilitate survival of cancer cells. Nevertheless, the relationship between alcohol intake and OS incidence had not yet been reported. Further studies are needed to illuminate the interaction between ALDH1B1 and oncogenic function involved in modulating tumor cell survival.

Furthermore, accumulating evidence suggests that cancer stem cell theory partially explains tumor progression and drug resistance and CSCs may contribute to chemoresistance by recreate the cellular heterogeneity of the parental tumor and [13, 41-43]. As a number of ALDH1 family, ALDH1A1 was linked to drug resistance in chemotherapy through regulating CSCs [29, 30, 35]. The present study also showed that ALDH1B1 silencing could suppress the protein expressions of cancer stem cell markers. Additional, we determined whether ALDH1B1 knockdown could enhance the cytotoxic effect of chemotherapeutic agent. In our study, we found that the inhibition of ALDH1B1 expression could increase the sensitivity of OS cells to chemotherapy (doxorubicin), suggesting that ALDH1B1 could be served as a therapeutic target to reverse chemotherapy resistance and combination therapies could be explored in osteosarcoma clinical trials.

\section{MATERIALS AND METHODS}

\section{Patient samples}

A retrospective study of 40 OS patients was enrolled. The data of age, gender, tumor sites, TNM stages, tumor size, metastasis and recurrence of patients were shown in Table 1. The study was approved by the human ethic committee of Henan Province Cancer Hospital. All patients provided written informed consent and the project was in accordance with the Helsinki Declaration of 1975. Their clinical information would be kept in the databases of Henan Province cancer hospital and utilized for research.

\section{Tissue microarray construction}

The OS TMA was constructed as described previously [3]. A retrospective study of 40 OS patients was identified for tissue microarray (TMA) immunohistochemical staining. To obtain representative 0.5-mm-diameter core biopsies, representative regions from haematoxylin and eosin-stained slides from each tissue block for each case were selected by one experienced pathologist. The TMA was constructed by the Servicebio company (Wuhan, China) using Quick-Ray manual tissue microarrayer set (UNITMA, Korea).

\section{Cell lines and cell culture}

The human OS cell lines (MG63, U2OS, and SAOS) were obtained from the American Type Culture 
Collection (Rockville, MD). The human osteoblast cells HOBC was purchased from PromoCell $\mathrm{GmbH}$ (Heidelberg, Germany). The human osteoblast cells HFOB was purchased from Cell Bank of Biomedical Sciences (IBS) of Shanghai Medical School (Shanghai, China). The OS cell lines were cultured in RPMI1640 medium (Gibco, USA) containing 10\% FBS (Gibco, USA), supplemented with $100 \mathrm{mg} / \mathrm{ml}$ streptomycin and $100 \mathrm{U} / \mathrm{ml}$ penicillin (Invitrogen, USA). All cells were maintained in a humidified atmosphere containing $5 \% \mathrm{CO}_{2}$ at $37^{\circ} \mathrm{C}$. All cell lines used in this study had been passed for less than 4 months in culture when the experiments were carried out. The cell lines used in this study are provided in Supplementary Table 1.

\section{Osteosarcoma GEO datasets process and analysis}

The set of microarray data (GSE21257, GSE39055) and corresponding clinical data were obtained from the GEO database (http://www.ncbi.nlm.nih.gov/geo). GSE21257 contained 53 OS samples and GSE39055 contained 37 OS samples. Expression data extraction was performed with R 3.2.5 software. Kaplan-Meier analysis were performed were analyzed by GraphPad Prism 5 software (San Diego, CA).

\section{Western blotting assay}

Total protein lysate from the OS cells was prepared with RIPA buffer (Beyotime, China) and quantified by BCA protein assay (Bio-Rad, CA). SPECTRAmax Microplate Spectrophotometer (Sunnyvale, CA) was applied to evaluate the protein concentrations. Then protein samples separated on SDS-polyacrylamide gels and transferred to PVDF membrane (Millipore, USA). After blocking in 5\% skimmed milk for 1 $\mathrm{h}$, the membranes were incubated with a primary antibody overnight at $4^{\circ} \mathrm{C}$ and following washed three times with TBST for $10 \mathrm{~min}$, then incubated with an HRP-conjugated secondary antibody for $1 \mathrm{~h}$ at room temperature. After washing 3 times for $10 \mathrm{~min}$ in TBST, the proteins were visualized with an ECL detection system (ECL; Amersham Pharmacia Biotech). The antibodies used in this study are provided in Supplementary Table 2 .

\section{Immunofluorescence}

Cells were resuspended in DMEM with 10\% FBS and plated onto coverslips for $24 \mathrm{~h}$. Then cells on coverslips were fixed in $4 \%$ paraformaldehyde, incubated with $0.3 \%$ Triton X-100-PBS for $15 \mathrm{~min}$, and blocked in 5\% goat serum, followed by incubating with
ALDH1B1 antibodies at $4^{\circ} \mathrm{C}$ overnight, and secondary antibodies for $1 \mathrm{~h}$ at room temperature (Boster, China). Hoechst 33258 (Beyotime, China) was used to stain the nuclei. Laser confocal scanning microscopy (Leica, Germany) was used to detect the immunofluorescent signals.

\section{Cell transfection and generation of ALDH1B1 stable knockdown cell lines}

Three shRNAs targeting ALDH1B1 gene (shALDH1B1-1, 2, 3) and negative control shRNA (sh-NC) with luciferase reporter were designed and synthesized by GenePharma (Shanghai, China). Cells were infected in six-well plates by shRNA lentiviruses and subsequently split into $10 \mathrm{~cm}$ dishes for selection over 72 $\mathrm{h}$ by using $2 \mu \mathrm{g} / \mathrm{ml}$ puromycin. The nonspecific siRNA (NC) and siRNA against ALDH1B1 were purchased from RiboBio (Guangzhou, China), and the transfection was carried out using lipofectamine ${ }^{\mathrm{TM}} 2000$ (Invitrogen, USA) as described previously [3]. Sequences of shRNA\&siRNA targeting ALDH1B1 and negative control shRNA were showed in Supplementary Table 3.

\section{MTT cell proliferation assay}

U2OS and SAOS were transfected with ALDH1B1 siRNA (45 nmol/L) or nonspecific siRNA (45 nmol/L). $5 \times 10^{3}$ cells in a volume of $100 \mu \mathrm{L}$ were seeded into a 96-well plate. Each group included three repeated wells. After 72 hours of incubation, proliferation assays were performed with MTT solution (Promega, USA) according to the manufacturer's protocol. The absorbance at a wavelength of $490 \mathrm{~nm}$ (A490) was measured on a SPECTRAmax Microplate Spectrophotometer from Molecular Devices (Sunnyvale, CA). All results were analyzed by GraphPad Prism 5 software (San Diego, CA).

\section{Invasion and wound healing assay}

Cell invasion assay was performed by using BioCoat Matrigel invasion chambers (BD, Germany) according the manufacturer's protocol. In brief, cells were transfected with ALDH1B1 siRNA or nonspecific siRNA in $0.5 \mathrm{ml}$ serum-free medium for $12 \mathrm{~h}$ and then seeded in upper chamber with a matrigel coated filter. Normal culture medium was used in lower chambers. The invading cells were fixed in 100\% methanol, stained in hematoxylin and counted under a microscope using a $200 \times$ objective (Nikon Instruments, Inc.).

The cell migration was determined by wound healing assay. Firstly, the cells were cultured in triplicate in 6-well plate with 50\% confluence. An artificial scratch was made by a $200 \mu l$ pipette tip and then monitored at 0 , $48 \mathrm{~h}$ using camera system (Nikon Instruments, Inc.). 


\section{Immunohistochemical (IHC) staining on TMA}

The expression of ALDH1B1 was determined based on the immunohistochemistry protocol (Paraffin) from Cell Signaling Technology (Beverly, MA) as previously described [44]. Briefly, the human OS tissue microarray sections were deparaffinized using xylene and rehydrated with graded alcohol, and then immersed in deionized water for 10 minutes. For antigen retrieval, the slides were immersed in boiling $\left(95-100^{\circ} \mathrm{C}\right)$ citrate buffer (pH 6.0) for $20 \mathrm{~min}$. After being blocked with $3 \%$ bovine serum albumin for $30 \mathrm{~min}$, sections were incubated with ALDH1B1 antibody at $4{ }^{\circ} \mathrm{C}$ overnight. The next day, the sections were incubated with corresponding peroxidase-labeled secondary antibody for $30 \mathrm{~min}$ at room temperature and washed with PBS for 3 times. Finally, Diaminobenzidine tetrahydrochloride (Boster, China) was used for the color-reaction and hematoxylin was used for nucleus counterstaining.

ALDH1B1 protein expression was assessed according to the extent and intensity of staining. The immunostaining intensity of ALDH1B1 was assessed independently by two scientists who had no knowledge of the clinical information, as follows: the staining extent in each core was scored as $1+(<25 \%), 2+(25-50 \%), 3+(50 \%$ to $75 \%)$, or $4+(>75 \%)$. Additionally, the staining intensity was quantified as 0 (negative), $1+$ (weak), $2+$ (intermediate), or $3+$ (strong). By multiplying the intensity and extension values, the final immunoreaction score was obtained (range $0-12$ ) and the samples were divided into five groups: $1+$ (score 0), 2+ (score 1-2), 3+ (score 3-4), 4+ (score 6-8), and 5+ (score 9-12), shown in Figure 1A. Meanwhile, for statistical purposes, patients in $1+, 2+$ and $3+$ groups were defined as low expression patients and in $4+$ and $5+$ groups as high expression patients. The images of ALDH1B1 staining for IHC and H\&E staining were obtained using the NanoZoomer 2.0-RS system (Hamamatsu Photonics Inc., Germany), and the digital slides were analyzed by the software of the NDP. view 2.5.14 version.

\section{Cell cycle and apoptosis analysis}

Cell cycle was analyzed by cell cycle kit (Beyotime, China) according to the manufacturer's instructions. Firstly, cells transfected with siRNA were plated in six-well plates for $48 \mathrm{~h}$. Then cells were collected and incubated with propidium iodide for $30 \mathrm{~min}$ in the dark. By flow cytometer (BD USA), cell cycle was analyzed and data were present as percentage distribution of cells in $\mathrm{G} 0 / \mathrm{G} 1, \mathrm{~S}$ and $\mathrm{G} 2 / \mathrm{M}$ phases of the cell cycle.

Apoptosis assay was performed with an Annexin V-APC antibody (Beyotime, China) and 7-AAD antibody (KeyGEN, China). Cells transfected with siRNA were plated in six-well plates for $48 \mathrm{~h}$. Then cells were harvested and washed three times in PBS. Cells were incubated in $300 \mu \mathrm{l}$ binding buffer added with $10 \mu 1$ of 7-AAD and
$5 \mu 1$ of Annexin V-APC for 15 min. Finally, cells were analyzed by flow cytometry ( 10,000 cells; BD USA).

\section{Chemotherapy drug sensitivity analysis}

The drug sensitivity analysis of OS cells was assessed by MTT assay. U-2OS, U-2OS/sh-NC, and U-2OS/shALDH1B1 cells were seeded into 96-well microplates at a density of $1 \times 10^{3}$ cells per well and incubated with a series of concentrations of anticancer drug doxorubicin. After 120 hours exposure to doxorubicin, proliferation assays were performed. Finally, the absorbance at a wavelength of $490 \mathrm{~nm}$ (A490) was measured on a SPECTRAmax Microplate Spectrophotometer from Molecular Devices (Sunnyvale, CA).

\section{In vivo tumor growth}

A total of 12 male athymic nude mice (4 to 6-week) were injected subcutaneously into bilateral flanks with either $5 \times 10^{6}$ the ALDH1B1 stably knockdown or negative control shRNA cell lines to create a tumor-bearing mice model of OS. Tumor growth was examined every 7 days for at least 28 days before the mice were sacrificed. Mice were photographed with an IVIS@ Lumina II system (Caliper Life Sciences, Hopkinton, MA) 10 minutes after an intraperitoneal injection of $4.0 \mathrm{mg}$ of luciferin (Gold Biotechnology, Inc., St. Louis, MO) in $50 \mu \mathrm{l}$ of saline. The tumor samples were fixed in paraffin for $\mathrm{H} \& \mathrm{E}$ and IHC staining. All the in vivo experiments were approved by the institutional animal care and use committee of Henan Province Cancer Hospital.

\section{Statistical analysis}

All of the statistical analyses were performed using the SPSS software version 23.0 (SPSS Inc., Chicago, IL). The data was presented as the means $\pm \mathrm{SD}$ from at least three separate experiments. The correlation of ALDH1B1 expression with clinicopathological characteristics in OS was performed by chi-squared test. Survival curves were analyzed by log-rank test. All differences were statistically significant at the level of $\mathrm{P}<0.05$.

\section{CONCLUSIONS}

In conclusion, our study demonstrates that ALDH1B1 is overexpressed and significantly correlated with poor prognosis of osteosarcoma patients. Moreover, ALDH1B1 is essential for osteosarcoma cell growth and survival in vitro and in vivo while promote apoptosis and cell cycle arrest in vitro. Furthermore, ALDH1B1 knockdown could enhance the cytotoxic effect of chemotherapy (doxorubicin) through regulating cancer stem cells in OS cells. Future studies to understand the molecular mechanisms of 
ALDH1B1 involved in cancer progression will be required. Our results suggest that ALDH1B1 can be considered as a potential prognostic marker and therapeutic target for OS patients, especially for patients with metastasis or drug resistant to chemotherapy.

\section{Ethics approval and consent to participate}

The study was approved by the human ethic committee of Henan Province Cancer Hospital. All patients provided written informed consent and the project was in accordance with the Helsinki Declaration of 1975. Their clinical information would be kept in the databases of Henan Province cancer hospital and utilized for research. All the in vivo experiments were approved by the institutional animal care and use committee of Henan Province Cancer Hospital.

\section{Consent for publication}

Not applicable.

\section{Availability of data and material}

Literature collection was performed by using electronic databases PubMed, Cochrane Library, and Web of Science. All statistical analyses were performed using SPSS 13.0 (SPSS, Chicago, IL, USA). Raw and processed data are stored in corresponding author of this paper and are available upon request.

\section{Abbreviations}

OS: osteosarcoma; ALDHs: aldehyde dehydrogenases; ALDH1: aldehyde dehydrogenase 1; CSCs: cancer stem cells; GEO: Gene Expression Omnibus; TMA: tissue microarray.

\section{Author contributions}

XW, YY, YTH, QCH and ZCT performed almost all the experimental work. XW, WWW and QQC participated in data analysis. STG, WTY, ZYL designed and performed the animal experiment. YGL and CL conceived the study and participated in its design. The manuscript was written by XW, RRS and YGL. All authors read and approved the final manuscript.

\section{CONFLICTS OF INTEREST}

The authors confirm that there are no conflicts of interest.

\section{FUNDING}

This study was supported by grants from the Henan medical science and technology breakthrough plan (No.201702256).

\section{REFERENCES}

1. Kansara M, Teng MW, Smyth MJ, Thomas DM. Translational biology of osteosarcoma. Nat Rev Cancer. 2014; 14: 722-35. https://doi.org/10.1038/nrc3838.

2. Bousquet M, Noirot C, Accadbled F, Sales de Gauzy J, Castex MP, Brousset P, Gomez-Brouchet A. Whole-exome sequencing in osteosarcoma reveals important heterogeneity of genetic alterations. Ann Oncol. 2016; 27: 738-44. https:// doi.org/10.1093/annonc/mdw009.

3. Li C, Chen Y, Bai P, Wang J, Liu Z, Wang T, Cai Q. LDHB may be a significant predictor of poor prognosis in osteosarcoma. Am J Transl Res. 2016; 8: 4831-43.

4. Mirabello L, Troisi RJ, Savage SA. International osteosarcoma incidence patterns in children and adolescents, middle ages and elderly persons. Int J Cancer. 2009; 125: 229-34. https://doi.org/10.1002/ijc.24320.

5. Anninga JK, Gelderblom H, Fiocco M, Kroep JR, Taminiau $\mathrm{AH}$, Hogendoorn PC, Egeler RM. Chemotherapeutic adjuvant treatment for osteosarcoma: where do we stand? Eur J Cancer. 2011; 47: 2431-45. https://doi.org/10.1016/j. ejca.2011.05.030

6. Khanna C, Fan TM, Gorlick R, Helman LJ, Kleinerman ES, Adamson PC, Houghton PJ, Tap WD, Welch DR, Steeg PS, Merlino G, Sorensen PH, Meltzer P, et al. Toward a drug development path that targets metastatic progression in osteosarcoma. Clin Cancer Res. 2014; 20: 4200-9. https:// doi.org/10.1158/1078-0432.CCR-13-2574.

7. Adamson PC, Blaney SM. New approaches to drug development in pediatric oncology. Cancer J. 2005; 11: 324-30.

8. Duchman KR, Gao Y, Miller BJ. Prognostic factors for survival in patients with high-grade osteosarcoma using the Surveillance, Epidemiology, and End Results (SEER) Program database. Cancer Epidemiol. 2015; 39: 593-9. https://doi.org/10.1016/j.canep.2015.05.001.

9. Zhou W, Hao M, Du X, Chen K, Wang G, Yang J. Advances in targeted therapy for osteosarcoma. Discov Med. 2014; 17: 301-7.

10. Zhao J, Chen F, Zhou Q, Pan W, Wang X, Xu J, Ni L, Yang H. Aberrant expression of microRNA-99a and its target gene mTOR associated with malignant progression and poor prognosis in patients with osteosarcoma. Onco Targets Ther. 2016; 9: 1589-97. https://doi.org/10.2147/ OTT.S102421.

11. Black W, Vasiliou V. The aldehyde dehydrogenase gene superfamily resource center. Hum Genomics. 2009; 4: 136-42.

12. Yang SM, Yasgar A, Miller B, Lal-Nag M, Brimacombe K, Hu X, Sun H, Wang A, Xu X, Nguyen K, Oppermann U, Ferrer M, Vasiliou V, et al. Discovery of NCT-501, a potent and selective theophylline-based inhibitor of aldehyde dehydrogenase 1A1 (ALDH1A1). J Med Chem. 2015; 58: 5967-78. https://doi.org/10.1021/acs.jmedchem.5b00577. 
13. Wu S, Xue W, Huang X, Yu X, Luo M, Huang Y, Liu Y, Bi Z, Qiu X, Bai S. Distinct prognostic values of ALDH1 isoenzymes in breast cancer. Tumour Biol. 2015; 36: 24216. https://doi.org/10.1007/s13277-014-2852-6.

14. Vasiliou V, Thompson DC, Smith C, Fujita M, Chen Y. Aldehyde dehydrogenases: from eye crystallins to metabolic disease and cancer stem cells. Chem Biol Interact. 2013; 202: 2-10. https://doi.org/10.1016/j.cbi.2012.10.026.

15. Pearce DJ, Taussig D, Simpson C, Allen K, Rohatiner AZ, Lister TA, Bonnet D. Characterization of cells with a high aldehyde dehydrogenase activity from cord blood and acute myeloid leukemia samples. Stem Cells. 2005; 23: 752-60. https://doi.org/10.1634/stemcells.2004-0292.

16. Ginestier C, Hur MH, Charafe-Jauffret E, Monville F, Dutcher J, Brown M, Jacquemier J, Viens P, Kleer CG, Liu S, Schott A, Hayes D, Birnbaum D, et al. ALDH1 is a marker of normal and malignant human mammary stem cells and a predictor of poor clinical outcome. Cell Stem Cell. 2007; 1: 555-67. https://doi.org/10.1016/j.stem.2007.08.014.

17. Balicki D. Moving forward in human mammary stem cell biology and breast cancer prognostication using ALDH1. Cell Stem Cell. 2007; 1: 485-7. https://doi.org/10.1016/j. stem.2007.10.015.

18. Shen X, Yue M, Meng F, Zhu J, Zhu X, Jiang Y. Microarray analysis of differentially-expressed genes and linker genes associated with the molecular mechanism of colorectal cancer. Oncol Lett. 2016; 12: 3250-8. https://doi. org/10.3892/ol.2016.5122.

19. Yokoyama Y, Zhu H, Lee JH, Kossenkov AV, Wu SY, Wickramasinghe JM, Yin X, Palozola KC, Gardini A, Showe LC, Zaret KS, Liu Q, Speicher D, et al. BET inhibitors suppress ALDH activity by targeting ALDH1A1 super-enhancer in ovarian cancer. Cancer Res. 2016; 76: 6320-30. https://doi.org/10.1158/0008-5472.CAN-16-0854.

20. Wang J, Mei F, Gao X, Wang S. Identification of genes involved in Epstein-Barr virus-associated nasopharyngeal carcinoma. Oncol Lett. 2016; 12: 2375-80. https://doi. org/10.3892/ol.2016.4940.

21. Liu X, Wang L, Cui W, Yuan X, Lin L, Cao Q, Wang N, Li Y, Guo W, Zhang X, Wu C, Yang J. Targeting ALDH1A1 by disulfiram/copper complex inhibits non-small cell lung cancer recurrence driven by ALDH-positive cancer stem cells. Oncotarget. 2016; 7: 58516-30. https://doi. org/10.18632/oncotarget.11305.

22. Flahaut M, Jauquier N, Chevalier N, Nardou K, Balmas Bourloud K, Joseph JM, Barras D, Widmann C, Gross N, Renella R, Muhlethaler-Mottet A. Aldehyde dehydrogenase activity plays a Key role in the aggressive phenotype of neuroblastoma. BMC Cancer. 2016; 16: 781. https://doi. org/10.1186/s12885-016-2820-1.

23. Çeliktaş M, Tanaka I, Tripathi SC, Fahrmann JF, Aguilarbonavides C, Villalobos P, Delgado O, Dhillon D, Dennison JB, Ostrin EJ. Role of CPS1 in cell growth, metabolism, and prognosis in LKB1-inactivated lung adenocarcinoma. J Natl Cancer Inst. 2016; 109: djw231.
24. You Q, Guo H, Xu D. Distinct prognostic values and potential drug targets of ALDH1 isoenzymes in non-smallcell lung cancer. Drug Des Dev Ther. 2015; 9: 5087-97.

25. Singh S, Arcaroli JJ, Orlicky DJ, Chen Y, Messersmith WA, Bagby S, Purkey A, Quackenbush KS, Thompson DC, Vasiliou V. ALDH1B1 as a modulator of pancreatic adenocarcinoma. Pancreas. 2016; 45: 117.

26. Matsumoto A, Arcaroli J, Chen Y, Gasparetto M, Neumeister V, Thompson DC, Singh S, Smith C, Messersmith W, Vasiliou V. Aldehyde dehydrogenase 1B1: a novel immunohistological marker for colorectal cancer. $\mathrm{Br}$ J Cancer. 2017; 117: 1537-43.

27. Shen JX, Jing L, Li GW, Huang YT, Wu HT. Mining distinct aldehyde dehydrogenase 1 (ALDH1) isoenzymes in gastric cancer. Oncotarget. 2016; 7: 25340-9. https://doi. org/10.18632/oncotarget.8294.

28. Hanahan D, Weinberg RA. Hallmarks of cancer: the next generation. Cell. 2011; 144: 646-74. https://doi. org/10.1016/j.cell.2011.02.013.

29. Wei Y, Wu S, Xu W, Liang Y, Li Y, Zhao W, Wu J. Depleted aldehyde dehydrogenase 1A1 (ALDH1A1) reverses cisplatin resistance of human lung adenocarcinoma cell A549/DDP. Thorac Cancer. 2017; 8: 26-32. https://doi. org/10.1111/1759-7714.12400.

30. Tanei T, Morimoto K, Shimazu K, Kim SJ, Tanji Y, Taguchi T, Tamaki Y, Noguchi S. Association of breast cancer stem cells identified by aldehyde dehydrogenase 1 expression with resistance to sequential Paclitaxel and epirubicinbased chemotherapy for breast cancers. Clin Cancer Res. 2009; 15: 4234-41. https://doi.org/10.1158/1078-0432. CCR-08-1479.

31. Marcato P, Dean CA, Giacomantonio CA, Lee PW. Aldehyde dehydrogenase: its role as a cancer stem cell marker comes down to the specific isoform. Cell Cycle. 2011; 10: 1378-84.

32. Luetke A, Meyers PA, Lewis I, Juergens H. Osteosarcoma treatment - where do we stand? A state of the art review. Cancer Treat Rev. 2014; 40: 523-32. https://doi. org/10.1016/j.ctrv.2013.11.006.

33. Chen R, Wang G, Zheng Y, Hua Y, Cai Z. Long non-coding RNAs in osteosarcoma. Oncotarget. 2017; 8: 20462-75. https://doi.org/10.18632/oncotarget.14726.

34. Bennett JH, Thomas G, Evans AW, Speight PM. Osteosarcoma of the jaws: a 30-year retrospective review. Oral Surg Oral Med Oral Pathol Oral Radiol Endod. 2000; 90: 323-32.

35. Ma I, Allan AL. The role of human aldehyde dehydrogenase in normal and cancer stem cells. Stem Cell Rev. 2011; 7: 292-306. https://doi.org/10.1007/s12015-010-9208-4.

36. Singh S, Arcaroli J, Chen Y, Thompson DC, Messersmith W, Jimeno A, Vasiliou V. ALDH1B1 is crucial for colon tumorigenesis by modulating Wnt/beta-catenin, notch and PI3K/Akt signaling pathways. PLoS One. 2015; 10: e0121648. https://doi.org/10.1371/journal.pone.0121648. 
37. Ma S, Chan KW, Lee TK, Tang KH, Wo JY, Zheng BJ, Guan XY. Aldehyde dehydrogenase discriminates the CD133 liver cancer stem cell populations. Mol Cancer Res. 2008; 6: 1146-53.

38. Deng S, Yang X, Lassus H, Liang S, Kaur S, Ye Q, Li C, Wang LP, Roby KF, Orsulic S. Distinct expression levels and patterns of stem cell marker, aldehyde dehydrogenase isoform 1 (ALDH1), in human epithelial cancers. PLoS One. 2010; 5: e10277.

39. Carpentino JE, Hynes MJ, Appelman HD, Zheng T, Steindler DA, Scott EW, Huang EH. Aldehyde dehydrogenase-expressing colon stem cells contribute to tumorigenesis in the transition from colitis to cancer. Cancer Res. 2009; 69: 8208.

40. Abdullah LN, Chow KH. Mechanisms of chemoresistance in cancer stem cells. Clin Transl Med. 2013; 2: 3.
41. Jordan CT, Guzman ML, Noble M. Cancer stem cells. N Engl J Med. 2006; 355: 1253-61. https://doi.org/10.1056/ NEJMra061808.

42. Kakarala M, Wicha MS. Implications of the cancer stemcell hypothesis for breast cancer prevention and therapy. J Clin Oncol. 2008; 26: 2813-20. https://doi.org/10.1200/ JCO.2008.16.3931.

43. Clarke MF. Self-renewal and solid-tumor stem cells. Biol Blood Marrow Transplant. 2005; 11: 14-6. https://doi. org/10.1016/j.bbmt.2004.11.011.

44. Zhang L, Guo S, Schwab JH, Nielsen GP, Choy E, Ye S, Zhang Z, Mankin H, Hornicek FJ, Duan Z. Tissue microarray immunohistochemical detection of brachyury is not a prognostic indicator in chordoma. PLoS One. 2013; 8: e75851. https://doi.org/10.1371/journal.pone.0075851. 